\title{
Economías morales del cuidado infantil. Familias, género y desigualdades en los programas de acogimiento familiar en la Argentina
}

\section{Moral economies of childhood care. Foster care policy and biological families}

\author{
Valeria Llobet ${ }^{1,11}$ \\ https://orcid.org/0000-0003-0673-8260 \\ vllobet@unsam.edu.ar \\ Carla Villalta ${ }^{\text {, III }}$ \\ https://orcid.org/0000-0003-4252-530X \\ carlavillalta@gmail.com \\ I Consejo Nacional de Investigaciones Científicas y Técnicas - Buenos Aires, Argentina \\ " Universidad Nacional de San Martín - San Martín, Buenos Aires, Argentina \\ II Universidad de Buenos Aires - Buenos Aires, Argentina
}




\title{
Resumen
}

En este artículo analizamos las principales características de los programas de "acogimiento familiar" implementados en Argentina, a fin de problematizar los modos en que las políticas participan en la configuración de afectos y emociones familiares y en la redistribución moral, generizada y enclasada de la responsabilidad sobre niños y niñas. A partir de datos construidos en una investigación empírica que caracterizó los programas estatales y no gubernamentales de acogimiento familiar desarrollados en el contexto nacional, procuramos problematizar los supuestos sobre el cuidado imbricados en ellos, las nociones acerca de la maternidad, paternidad y niñez privilegiadas y también cómo esas nociones se vinculan con las formas de organización social del cuidado y los sentidos de niñez desplegados por los sectores populares. Estos programas constituyen un analizador relevante en tanto permiten observar los procesos de redefinición de las relaciones entre estado, mercado y familias así como la producción de exclusiones y jerarquías de género.

Palabras clave: infancia; género; maternidad; cuidado.

\begin{abstract}
In this article, we analyze the principle characteristics of the "foster care" programs, with the aim of problematizing the modes in which policies participate in the configuration of family affections and emotions and in the moral, gender-based and class-based redistribution of the responsibility for children. Using data from an investigation on state and non-governmental programs developed in Argentina, we aim to problematize the assumptions about care interwoven in these programs, notions about maternity, paternity and childhood privileged and also how those notions connect to the forms of social organization of care and the feelings about childhood that are deployed by the working class sectors. As such, these programs make up a relevant subject to the extent that they allow us to observes the processes of redefinition of the relationships between the state, marketplace and families as well as of the production of exclusions and gender hierarchies.
\end{abstract}

Keywords: childhood; gender; maternity; care. 


\section{Introducción}

Las modalidades del cuidado que debe ser provisto a los niños y niñas que por diferentes razones se encuentran separados de su medio familiar, es uno de los puntos más álgidos en las discusiones sobre las políticas implementadas en relación con la protección de la infancia (Bittencourt Ribeiro, 2012; Fonseca, 2009; Uriarte Bálsamo; Fonseca, 2009; Villalta; Rojas; Gesteira, 2019). Las formas que ese cuidado debe asumir, los lugares institucionales en los que se lleva a cabo, los plazos adecuados para su desarrollo, así como los agentes que lo deben desplegar han sido cuestiones sumamente controversiales en el campo de organismos destinados a la protección de niños, niñas y adolescentes. De este modo, los sentidos que se movilizan en diseños y prácticas institucionales permiten vislumbrar específicos regímenes morales a partir de los cuales se piensa la niñez, sus necesidades y derechos así como el lugar asignado a la "familia" como dispositivo esencial para su adecuado crecimiento y desarrollo. En este artículo, nuestro interés es focalizar en las principales características de los programas de acogimiento familiar que en los últimos años comenzaron a ser implementados en la Argentina. Estos programas son pensados como "antagónicos" al cuidado de tipo residencial ya que valorizan a la familia como el espacio más adecuado para un niño o niña y enfatizan el valor del cuidado singularizado y la atención personalizada en tanto requisitos para el correcto y pleno desarrollo de la subjetividad y autonomía progresiva de niños y niñas. Este tipo de cuidado, al emular a "la familia" tanto reproduce y reactualiza, como problematiza y tensiona las nociones acerca de la maternidad y paternidad adecuadas, del afecto familiar y la calidad del cuidado provisto, las obligaciones asociadas a las diferentes categorías de parientes, la distribución generizada de las tareas domésticas, entre otras (Cadoret, 1995, 2006).

Las reflexiones que presentamos aquí se basan centralmente en los datos producidos a partir de un trabajo de relevamiento, sistematización y análisis de programas "de cuidado alternativo en ámbito familiar" que hemos realizado para Unicef-Argentina, durante los años 2017-2018 (Llobet; Villalta, 2018). ${ }^{1}$

1 La investigación que coordinamos supuso la realización de un trabajo de campo intensivo, en 13 jurisdicciones de la Argentina, a partir del cual se realizaron un total de 155 entrevistas en profundidad a coordinadores y profesionales de los programas de Acogimiento Familiar $\rightarrow$ 
En esa indagación, desarrollada a través de un enfoque cualitativo y un intensivo trabajo de campo, analizamos las modalidades, usos y sentidos que rodean a los programas de acogimiento familiar en distintas localidades de la Argentina e identificamos las tensiones y dilemas más recurrentes que genera su implementación. Asimismo, el trabajo que presentamos aquí se enmarca en una discusión más amplia sobre las transformaciones en la mater-paternidad, la crianza y las nociones de infancia derivadas de la institucionalización local del discurso transnacional de derechos de la niñez tanto en el campo de organismos y agentes de protección de la infancia como en las políticas sociales (Llobet; Villalta, 2019), en un contexto de aumento de la pobreza y de dominancia neoliberal, que ha sido denominado como crisis de la reproducción social (Federici, 2018). En este artículo nos preguntamos ¿cuáles son los supuestos sobre el cuidado imbricados en las previsiones para las tomas de las medidas de protección de derechos de los niños y niñas? ¿Qué mater-paternidades y niñeces son privilegiadas por los sentidos institucionalizados en prácticas y normas? ¿Cómo las políticas participan de la configuración de los afectos y emociones familiares, y de la redistribución moral, generizada y enclasada, de la responsabilidad por los niños? (Fonseca, 2002; Lugones, 2012; Stoler, 2007; Villalta, 2010; Zelizer, 1994) ¿Cuál es la capacidad que tienen las familias de origen de los niños en este tipo de programas de cuidado alternativo para expresar sus demandas y necesidades?

Las políticas de protección de derechos cada vez más se han concentrado en la regulación de las prácticas familiares de cuidado y crianza (Barna, 2015; Darré, 2013; Llobet, 2009; Magistris, 2016; Villalta, 2010) en un proceso que fue

$\rightarrow$ (gubernamentales o dependientes de OSC); a Coordinadores y profesionales de Organismos administrativos de protección de derechos; a autoridades de las áreas de Niñez (provincial y municipal); a Jueces/zas de familia, a Asesores Tutelar, a Equipos Técnicos de Juzgados de Familia; y a integrantes de familias acogedoras y en un caso a una familia de origen. Los datos primarios producidos a partir de las entrevistas buscaban relevar dimensiones descriptivas de los programas -historia, recursos, procedimientos, modalidades de trabajo-y de la población asistida -situación jurídica y características generales de las/os niños/as y de las familias acogedoras, entre otros. Asimismo, relevamos y sistematizamos material documental y estadístico sobre las y los niños/as sin cuidados parentales y los programas de acogimiento. El estudio fue promovido por la Red Federal de Acogimiento Familiar (creada en 2014) y financiado por Unicef-Argentina. El equipo de investigadores estuvo conformado por: Agustín Barna, Silvina Fernández, Ana Cecilia Gaitán, Soledad Gesteira, Julieta Grinberg, Gabriela Magistris, Florencia Paz, Soledad Rojas Novoa, Adrián Rozengardt. 
denominado como familiarismo (Haney; Pollard, 2003) o maternalismo (Nari, 2004). En tal proceso, altamente generizado y enclasado, algunos autores hallan que las tendencias proteccionistas globalizadas hacen de los derechos unos instrumentos eventualmente negativos para los propios niños (Cheney, 2013), dado que enmascaran la pobreza estructural y la injusticia social mediante la moralización de las desigualdades sociales (Fassin, 2009) y movilizan de maneras contradictorias las necesidades y derechos de las mujeres y de niños y niñas (Llobet, 2014) o crean nuevas oposiciones que resultan empobrecedoras para ambos (Rosen, 2019). Así, se ha señalado que la lógica contemporánea de gobierno a través de los derechos de la niñez reproduce las jerarquías de proyectos de gobierno anteriores, a la vez que legitima nuevas exclusiones en el marco de articulaciones neoliberales (Balagopalan, 2019) en las que la familia pobre es un sitio de gobierno (Rosen, 2019) y el individuo es responsable por su reproducción.

No obstante, también es posible señalar el carácter ambiguo de la retórica de derechos ya que si bien puede incrementar la regulación de las poblaciones de sectores populares, también potencialmente permite viabilizar la incorporación de sus demandas y, de tal modo, aportar una vía de ampliación de los sentidos de justicia (Villalta, 2010). Así, el discurso de derechos es un lenguaje legítimo y disponible que sectores previamente "minorizados" o portadores de "ciudadanías subordinadas" pueden movilizar para obtener formas de legitimación y de politización de necesidades. Aquí procuramos contribuir a este debate, a partir de visibilizar la micropolítica de la "protección de derechos" en relación con el cuidado de niños y niñas atendiendo a la naturaleza generizada de la responsabilización neoliberal (Rosen, 2019).

Para ello, este artículo analiza y problematiza las principales características que ha asumido la implementación del acogimiento familiar en la Argentina, y se organiza en cuatro partes. En la primera, inscribimos el surgimiento de este tipo de iniciativas en un proceso socio-histórico signado por la familiarización del cuidado, la revaloración de la dimensión afectiva y la psicologización de la crianza. En el segundo apartado, describimos los principales rasgos de los programas de acogimiento familiar que hemos relevado y caracterizado en nuestra investigación, y analizamos algunas de sus características más salientes, entre ellas, la preferencia por familias "ajenas" a la red vincular de los niños para que desarrollen" el acogimiento", el escaso margen que les queda a las familias 
de origen de los niños y niñas para resolver arreglos relativos a la crianza de sus hijos, la falta de contacto entre familias de acogimiento y de origen, entre otras. En la tercera parte, caracterizamos las familias que participan en este tipo de programas y problematizamos el notorio sesgo generizado y clasista que la mayoría de estas iniciativas ha asumido. En la última parte, a partir de examinar otras tensiones -entre ellas, afecto y cuidado, cuidado y dinero, público y privado- reflexionamos sobre los modos en que ellas tiñen de particulares características la práctica cotidiana de los programas destinados a la protección de niños, niñas y adolescentes.

\section{Las modalidades institucionales del cuidado de la niñez}

Las transformaciones socioculturales que dotaron a la familia -heterosexual, conyugal y de sectores medios- de centralidad en el bienestar psi de niños y niñas, ${ }^{2}$ a partir de la emergencia de un modelo psicologizado de crianza (Cosse, 2010; Fonseca, 1998) también condujeron, a partir de la década de 1960, a la creación de programas de asistencia basados en la imitación del hogar familiar. Bajo la forma de pequeños hogares y familias sustitutas, las intervenciones de reemplazo de las familias inadecuadas se concebían como las estrategias de preferencia, sobre todo para atender a la "delincuencia juvenil", el "maltrato" y el "abandono", incluso si su extensión y capacidad objetiva nunca alcanzó la masividad que su relevancia discursiva hubiera requerido. Hacia la década de 1990, los debates internacionales que condujeron a la sanción de la Convención de los Derechos del niño y los nuevos discursos de pobreza infantil, interpelaron a la familia como "responsable natural" y primaria en la garantía del acceso a derechos y sitio privilegiado de intervención para las políticas anti-pobreza de los Estados neoliberales (Llobet, 2009). Desde el punto de vista de los actores promotores de la protección de la niñez, "la familia" se construyó como un lugar idealizado de cuidado y protección en contraposición a

2 Modelo de parentalidad cuyo énfasis se halla en una relación emocional cercana entre padres e hijos, la democratización de las relaciones intrafamiliares propiciadas por el feminismo (Barrancos, 1999; Nari, 2004), y la transformación de la estructura económica que requirió de la progresiva e intensiva empleabilidad de las mujeres y la postergación de la maternidad (Jelin, 1998; Torrado, 2003) en los sectores medios y trabajadores urbanos. 
la institucionalidad fría y anónima del estado entendido como equivalente a una "institución total". De esta manera, a partir de los cuestionamientos a las intervenciones abusivas y arbitrarias sobre las familias pobres, se abonó el camino de la psicologización y afectivización de la mater-paternidad. En otras palabras, la crítica a la institución total y al trato despersonalizado de los niños se basó en una reivindicación de la "familia" que fue construida como el entorno ideal y principal para la garantía de derechos de niños y niñas y, a la vez, la responsabilidad de madres y padres se configuró como un imperativo moral personal (Balagopalan, 2019). Estas regulaciones sobre las familias no solo se efectivizaron mediante disposiciones legales vinculadas con el ordenamiento de las relaciones, sino también desde la política social (Commaille; Martín, 1998).

En efecto, no es novedad que las políticas de protección a la niñez se han dirigido históricamente a reformar y civilizar a las familias de sectores populares, sobre la base de juicios morales, científicos, emocionales que las hacían responsables de su situación y del desorden social. Ahora bien, para autoras como Vianna (2002), tal moralización al menos en Brasil -lejos de adoptar el modelo tutelar que plantean análisis como los de Donzelot (1990) para Francia- administra estas poblaciones negociando la "mejor solución posible" y considerando de manera singular y tentativa "lo mejor para el niño", a la luz de una economía moral en la que lo intolerable en torno de la infancia es construido a partir de las negociaciones prácticas, lo dicho y no dicho, y las posibilidades de complemento de la autoridad estatal y la autoridad parental. La producción social de la guarda de los menores (Vianna, 2002) es así atravesada por múltiples negociaciones entre lo ideal y lo posible (Villalta, 2012), y por las tensiones en torno a la redefinición de la responsabilidad estatal sobre las familias (Souza Lima; Macedo e Castro, 2015).

Los procesos de reubicación y guarda de niños como estrategia de protección de derechos buscan crear, formalizar y ordenar relaciones familiares están informados por una economía moral de los derechos de la niñez que a la vez configuran. En especial, las prácticas de reubicación de niños y niñas, la inscripción y des-inscripción de los niños en relaciones familiares y filiatorias, constituyen estrategias de ordenamiento a través de las cuales se procura materializar la "protección de los derechos la niñez" y en ese sentido son productivas de específicos sentidos sobre lo que tal protección debe ser. La formalización 
burocrática de los arreglos de cuidado en las redes familiares -por ejemplo, mediante las guardas- junto con el establecimiento de plazos cada vez más ajustados para resolver sobre la adopción o la revinculación de niños/as separados de su medio familiar (Villalta, 2015), conduce a que las medidas de protección tiendan a basarse en la modificación del emplazamiento de los niños en las redes de relaciones familiares y sociales, a la vez que promueven el reordenamiento de los arreglos familiares informales. Niños y relaciones familiares son formas de capital emocional a la vez que recursos.

En el marco del desarrollo de este tipo de estrategias, Fonseca halló que la noción de "familia maltratadora" configura una categoría clasificatoria central para el ordenamiento de una intervención que, en lugar de corregir las condiciones que provocaron el retiro de los niños, tiende a exacerbarlas (Fonseca, 2002) y a tornar la reubicación transitoria en definitiva. La colocación de niños y niñas en diferentes entornos familiares es una práctica de larga data en la creación y transformación de las relaciones familiares, en especial en América Latina (Villalta, 2012). La formalización de estas prácticas en los últimos años se ha incrementado, Convención de Derechos de la Niñez mediante, a través de distintos institutos jurídicos y programas que se vinculan tensamente con las prácticas familiares de los sectores populares. ${ }^{3}$ Es en este marco de formalización, que los programas de acogimiento familiar son promovidos como un modelo ideal/izado de intervención transitoria.

\section{El Acogimiento Familiar en Argentina}

En las últimas dos décadas, de la mano de numerosos organismos y redes regionales e internacionales, las políticas de acogimiento familiar, esto es, de "cuidado alternativo en ámbito familiar", se han configurado como las políticas

3 La disyunción entre las leyes y las experiencias de sectores populares ha sido analizadas en tanto implicadas en la reproducción de la desigualdad (Thompson, 2010). En especial, las transformaciones de las normas que regulan el parentesco y la crianza han sido analizadas como eficaces mecanismos de poder y en tanto productoras de brechas que invisibilizan o bien penalizan arreglos familiares de los sectores populares (Fonseca, 2002; Leinaweaver, 2009; Milanich, 2002; Villalta, 2011). 
de preferencia para resolver la situación de niños y niñas "privados de cuidados parentales". La mayoría de los países de América Latina han desarrollado programas de acogimiento familiar que buscan reemplazar la institucionalización de niños y niñas a cargo de la tutela del Estado. ${ }^{4}$

En la Argentina, estos programas han sido implementados en la mayoría de las jurisdicciones del país, de hecho solamente tres provincias del total de veinticuatro no tenían programa en 2018. Se trata de programas estatales que, en algunos casos, firman convenios con ONG especializadas en la temática. La mayor parte se ha creado recientemente y se encuentra inspirada en los instrumentos internacionales más recientes sobre cuidado alternativo de niños, niñas y adolescentes, en especial en las Directrices sobre Modalidades de Cuidado Alternativo de la ONU, aprobadas en 2009. En general, se componen de un equipo técnico formado por psicólogos y trabajadores sociales, quienes tienen por función seleccionar a las familias, supervisarlas y acompañarlas mientras dure el acogimiento de un niño o niña.

Más allá de estar creados en casi todas las provincias del país, estos programas atienden a un número ínfimo de niños y niñas, alrededor del millar anual, un escaso 10\% de las medidas de protección especial de derechos, y la cantidad de familias - previamente seleccionadas- que se desempeñan en ellos eran aproximadamente 700. Las razones de su limitado impacto se vinculan -tal como hemos podido advertir a partir del desarrollo del trabajo de campo realizado en distintas localidades del país- tanto con los sentidos sociales locales en torno a la adopción y los lazos familiares, como a decisiones de diseño, derivadas de las condiciones de posibilidad construidas en torno a la colocación de niños en familias "ajenas". Las dos principales: el temor a recrear posibilidades de "apropiación de niños", cuestión que adquiere relevancia en nuestro país al conectar con las prácticas criminales de apropiación ocurridas durante la última dictadura militar (Villalta, 2012), como tradicionales derivadas de la externalización clasista de la reproducción (Marre, 2009). La segunda,

4 Este tipo de intervenciones no es novedosa, y sus raíces se extienden hasta los inicios del siglo XX con las políticas de "colocación familiar" de niños que se encontraban en los establecimientos de la asistencia pública. Promediando el siglo XX distintas iniciativas se implementaron bajo el nombre de "familias sustitutas". 
el escándalo que rodeó la terminación del tradicional programa de Amas Externas en la década de 2000, escándalo vinculado con la mercantilización del cuidado, el manejo de fondos y la sordidez y violación de derechos que acompañaba el trato cotidiano a niños/as. ${ }^{5}$

Estas características de los programas tienen dos grandes consecuencias. La primera, que el acogimiento familiar se utiliza para implementar medidas de protección especial de derechos, que se toman por un plazo máximo de 180 días. Al avanzar en la formalización de la medida (en tanto en toda separación de niños/as de su medio familiar debe mediar la intervención institucional) y en la rigidez de la temporalidad (180 días como máximo) a la vez que se busca evitar la eternización de niños y niñas en situaciones diseñadas como transitorias o "limbos", también se contribuye -en algunos casos- a restringir la posibilidad de acuerdos informales gestionados por las familias y de usos diversos de los programas de cuidado alternativo por parte de los parientes de los niños. Esto no quiere decir, por supuesto, que las familias no continúen gestionando conflictos y recursos mediante la "circulación de niños y niñas" (Fonseca, 1998; Leinaweaver, 2012; Llobet, 2019), sino que, en la medida en que tales situaciones son legibles para el estado y el arreglo es considerado vulneratorio de derechos por los agentes intervinientes, las burocracias especializadas las administran reduciendo la agencia de madres, abuelas y/o de otros familiares que eventualmente necesiten o quieran ceder transitoriamente el cuidado de niños y niñas para hacer frente a su crianza. Ello también afecta la agencia

5 El Servicio de Amas Externas fue creado en la órbita nacional en el año 1969, y tenía por objetivo proveer cuidado transitorio a niños/as hasta los 5 años, a cargo de una mujer seleccionada y contratada al efecto, que podía acoger desde 2 hasta 4 niños/as en forma simultánea. A este programa se sumó en 1980 el servicio de Amas Externas de Cuidado Preferencial, para niños/as de hasta 3 años con "padecimientos o patologías psicofísicas". Con los años, esta modalidad de cuidado fue duramente cuestionada, y en 2005 una vez que se sancionó la ley 26.061 de Protección Integral de derechos de niños, niñas y adolescentes, y se creó la Secretaría Nacional de Niñez, Adolescencia y Familia (en reemplazo del antiguo Consejo Nacional de Niñez, Adolescencia y Familia), dejó de existir. Las críticas fundamentalmente se dirigían a la calidad del cuidado provisto por las Amas quienes podían alojar en sus casas alrededor de 8 niños/as a la vez; ya que el cuidado personalizado y singular que se había perseguido con su instrumentación se había diluido, y las amas alojaban simultáneamente cada vez a más niños/as. Esta práctica además era interpretada como un indicio del afán de lucro de estas cuidadoras, ya que cobraban una beca por cada niño/a que alojaban (Villalta; Rojas; Gesteira, 2019). 
de los propios niños, niñas y adolescentes que ven recortada su posibilidad de decidir convivir con otra familia (familias vecinas, de amigos, de novios/as) o en arreglos autónomos, en tanto esa situación formalmente debería ser enmarcada en los estrechos marcos que supone una medida de protección excepcional de derechos. Esto es, en un acto administrativo que toman los agentes de los organismos de protección de derechos -y controlan en su legalidad los juzgados de familia- por el cual se formaliza la separación de un niño/a de sus progenitores por un lapso máximo de seis meses.

Asimismo, la mayoría de los niños y adolescentes que son separados de su medio familiar son derivados a los denominados hogares convivenciales, salvo en casos como las provincias de San Luis, Neuquén y Tierra del Fuego o en municipios como San Martín, que privilegian las medidas con familia ampliada propia del niño/a o con familia de acogimiento (Unicef; Secretaría Nacional de Niñez, Adolescencia y Familia, 2018).

La segunda consecuencia es que se privilegia el acogimiento en familias "externas". Esto es, en familias que no han tenido contacto ni vínculo previo con los "casos" y que han sido preseleccionadas por el programa y conforman un "banco" estable de familias, o bien son parte de una red de recursos institucionales, tanto de ONGs o de iglesias, como de redes más o menos informales del funcionariado. Como condición sine qua non, las familias no deben estar inscriptas en el Registro Único de Aspirantes a guardas con fines de Adopción (RUAGA), a excepción de dos jurisdicciones (San Luis y Corrientes) y en la mayoría de los casos en que se cuenta -o se aspira a contar-con un banco de familias preseleccionadas, se realiza un proceso de evaluación y de admisión psico-social para tales familias. La mayoría de los programas no incluye a las familias extensas o con vínculo previo con niños y niñas. Solo algunas jurisdicciones no establecen distinciones entre familias "ajenas", "ampliada" y referentes comunitarios. Estas distinciones revisten relevancia para delimitar el tipo de relación que establece el programa:

Acogimiento es un programa donde hay un antes y un después del ingreso de un chico. Si yo tengo una medida excepcional con un referente comunitario eso no es acogimiento; es más, ese referente puede llegar a pedir eventualmente una guarda de ese niño. (Entrevista realizada a funcionaria del Área de Niñez de la provincia de Entre Ríos, noviembre de 2017). 
De los diecinueve programas provinciales existentes en el momento en que realizamos el trabajo de campo, la mayoría trabaja solamente con las llamadas "familias ajenas", cuatro programas también trabajan con familias de la comunidad, y uno con las familias extensas de los niños/as, mientras que tres programas se encontraban en proceso de implementación y no especificaron con qué tipo de familia trabajarían.

Idealmente estos programas se deben acompañar de medidas de apoyo a las familias de origen para "desarrollar adecuadamente sus funciones de cuidado", ya sea mediante recursos materiales y/o diferentes formas de apoyo tales como gestión de turnos para la asistencia en salud mental, subsidios para resolver situaciones de extremo hacinamiento en el hogar, etc. Sin embargo, en ninguna jurisdicción se relevó la existencia de programas específicos de fortalecimiento a la familia de origen. ${ }^{6}$ Así, los recursos destinados al fortalecimiento familiar son escasos en la mayoría de las localidades, consistiendo centralmente en los mismos que maneja el Organismo Administrativo para cualquier intervención, y no disponen de previsiones administrativas para apoyar económicamente a las familias de origen. Así, cuando la medida excepcional de protección de derechos se instrumenta en la familia extensa de los niños/as o incluso en el entorno comunitario, rara vez es acompañada de recursos específicos. La tramitación de la Asignación Universal por Hijo (AUH) -transferencia condicionada de ingresos por niño $0^{7}$ para el nuevo cuidador es una obligación normativa. No obstante, en la práctica se concreta sólo si los agentes consideran la situación como permanente, viable y legítima. Además, una vez retirado el niño

6 Si bien en algunas localidades como la Ciudad de Buenos Aires, existen programas de fortalecimiento de vínculos o fortalecimiento familiar, se trata de programas que movilizan esencialmente recursos de acompañamiento a las familias previamente a la toma de una medida excepcional de protección de derechos.

7 En el año 2009, a partir de la implementación de la Asignación Universal por Hijo (AUH) se avanzó en la dirección de extender el sistema de asignaciones familiares a los grupos familiares desempleados, con empleos precarios e informales o con ingresos mínimos. A diferencia de las TCI "clásicas", por ejemplo los programas "Bolsa Família” brasileño y “Oportunidades" mexicano, que consisten en programas transitorios que aportan una transferencia monetaria a hogares seleccionados mediante tecnologías de focalización, requiriendo un conjunto de contrapartidas, que se asocian a logros en capital humano, la AUH tiene una transferencia dineraria y algunas condicionalidades, pero no es "transitoria", y técnicamente no es focalizada, aún cuando sea discutible su carácter "universal". Conceptualmente, extiende el régimen de protección social a las familias asociado al trabajo formal (asignaciones familiares). 
mediante una medida de protección excepcional, los funcionarios gestionan que las familias de origen pierdan el recurso de la AUH equivalente a la cantidad de niños/as bajo medida.

$\mathrm{Si}$ bien la normativa es clara en la prioridad dada a las familias de origen y ampliada, no especifica qué tipo de apoyos se movilizarán para lograr la revinculación. Solo se estipula la realización de visitas o entrevistas supervisadas que muchas veces terminan desalentando -a través de microprácticas disuasorias (Ciordia; Villalta, 2012)- a las familias de origen de los/as niños/as en tanto son ocasión de demostrarles todo aquello que "no pueden" o "no cumplen". Lo estipulado normativamente y los protocolos de actuación parecen así funcionar muchas veces como obstáculos para la revinculación con las familias de origen:

A veces pienso que somos muy estructuradas en el proceso, con el legajo, las relaciones que se establecen con los niños, los procedimientos y burocracias. Conozco otras experiencias que son más flexibles. El problema es cuando hay un problema y los recorridos no están claros o hay mucha informalidad. (Entrevista realizada a una profesional del equipo técnico del Programa de Acogimiento Familiar, Ciudad de Cipolletti, Río Negro, noviembre de 2017).

Al mismo tiempo, los programas de acogimiento familiar que hemos relevado no prevén el trabajo con la familia de origen, sino que en la mayoría de los casos es la misma área que tomó la medida excepcional, es decir, los agentes que retiraron al niño quienes continúan trabajando con su familia en aras de la restitución. Complementariamente, el contacto entre la familia de origen y la familia acogedora es evitado por parte de los funcionarios y trabajadores. Esto es, en ningún caso las familias de origen conocen el domicilio en el que reside su hijo/a y en la mayoría de las jurisdicciones, los programas de acogimiento no promueven el contacto de los niños con sus familias de origen o bien lo desalientan para proteger la intimidad de la familia acogedora. Relatos sobre la conflictividad de las familias de origen, la incomodidad de la familia acogedora en un encuentro fortuito, y las referencias más o menos explícitas a intentos de recuperación de los niños por parte de las madres puntúan las explicaciones dadas por las y los trabajadores de los programas para señalar porqué se evita tal contacto. 
El desarrollo de este tipo de prácticas parece contribuir al despliegue de estrategias que se vinculan con la producción social de "niños adoptables" (Leinaweaver, 2009) y la estratificación de la reproducción. Además, a través de sutiles mecanismos reintroduce y refuerza relaciones de desigualdad entre familias de acogimiento y familias de origen (Fonseca, 2002). Cuestión que también resulta visible si analizamos, tal como hacemos en el próximo apartado, los sentidos que adoptan las modalidades de gestión del acogimiento por parte de las familias de acogida.

\section{Cuidados y modelos de familia en tensión}

$\mathrm{Al}$ examinar las principales características que tienen los programas de Acogimiento Familiar que se han desarrollado durante los últimos años en las distintas jurisdicciones de la Argentina, resulta notorio el sesgo generizado y clasista que han adquirido en su implementación. En algunos casos, esa impronta se vincula con la pertenencia a iglesias católicas y evangélicas de las familias seleccionadas y/o de las ONG's que desarrollan esos programas en convenio con los gobiernos locales. Así, los sentidos vinculados a la caridad, el altruismo, la solidaridad, la espiritualidad se entrelazan, y configuran poderosas razones para explicar la tarea desarrollada. Como una profesional del equipo técnico de uno de los programas provinciales mencionara en la entrevista que le realizamos, es usual que quienes quieren desempeñarse como familia de acogimiento expresen que su motivación para hacerlo se vincula a la necesidad de "ganarse un pedazo de cielo".

A su vez, al analizar las maneras en que el cuidado de los niños/as es concretamente realizado en las familias de acogimiento, es notorio que éste es desarrollado según esquemas basados en una distribución de género tradicional. Como señalaron varios entrevistados, usualmente son mujeres, que en ocasiones cuentan con la asistencia de hijos mayores, quienes tienen a su cargo la mayor parte de las tareas de cuidado y crianza. Además, en la medida en que mayoritariamente los/as niños/as derivados a acogimiento tienen hasta 3 o 4 años, es raro que sean escolarizados o incorporados en jardines maternales o guarderías, de modo que la mayor parte del tiempo el cuidado se realiza en el hogar de la familia de acogimiento. Debido a su extracción de clase y en no 
pocos casos a su pertenencia a iglesias, ${ }^{8}$ las familias cuentan con redes sociales que les permiten movilizar recursos de apoyo, redes que son valoradas por los agentes institucionales y contribuyen a la propia selección de la familia. Así, las mujeres que realizan el acogimiento no padecen el aislamiento social o el confinamiento a las redes familiares que es característico de la experiencia maternal de las mujeres de sectores populares (Llobet; Milanich, 2014), mayoritariamente proveedoras de los niños y niñas que serán acogidos.

Tal como se encuentran diseñados los programas de acogimiento familiar, las cuidadoras no cuentan con apoyos para limitar o restringir el trabajo remunerado -de existir tal necesidad- dado que está expresamente prohibido que perciban una remuneración por las tareas de cuidado. Ello implica una sobre-representación de familias con mujeres fuera del mercado laboral, con una inclusión en el mismo a tiempo parcial, o bien con más de dos miembros con capacidad de brindar cuidados (hijos adolescentes o jóvenes convivientes) de modo que el acogimiento es, como señalaron numerosos entrevistados, un "proyecto familiar". El carácter "solidario" y no remunerado de esta tarea es un rasgo especialmente valorado por el diseño del programa y por los agentes:

Donde empiezan a darle dinero por cada niño, esto termina mal porque empieza a ser un trabajo. Y en una época como esta en la cual hay muchas dificultades económicas no estaría bueno. Nosotros ya te digo, hacemos milagros, conseguimos ropa, conseguimos calzado, todo lo que se pueda conseguir, pero no le ponemos un monto fijo. Los remedios, vos podés contar, si el chico se enferma y desde [nombre de la OSC], no importa desde dónde, aun sea de nuestros propios bolsillos, se cubre todo lo que necesita. (Entrevista a profesional de un programa no gubernamental de Acogimiento Familia, noviembre de 2017).

Lo planteamos solidario, sin transferencia de recursos, solo un apoyo para lo alimentario y los gastos básicos de cuidado. Cuando eran 'Familias Sustitutas'

8 Algunos de los programas de Acogimiento Familiar estatales se han instrumentado a partir de la realización de convenios con ONG's y/o con iniciativas llevadas adelante por organizaciones laicas católicas o por iglesias evangélicas. En este sentido, los valores y sentidos asociados a la caridad y solidaridad permean la dinámica del cuidado que proveen estas organizaciones y las familias de acogimiento se ven apoyadas también por redes comunitarias propias de su pertenencia a la organización eclesial. 
(denominación anterior) se priorizó lo económico antes que lo solidario, entonces terminamos con familias que solo venían a cobrar un sueldo. Ahí sí había una cantidad de familias. Hoy nos juega en contra la falta de trabajo, la falta de estabilidad, la falta de contención económica de los grupos familiares, la falta de políticas públicas en cuanto a salario digno, en este contexto la solidaridad tiene sus límites. (Coordinador de Programa estatal de Acogimiento Familiar, Entre Ríos, noviembre de 2017).

También es muy común, hay mucha Familia Solidaria de gente clase media ahí, hay mucha generosidad en las clases media más ajustada, entonces, insisto no me parece que sea un trabajo que haya pagar, pero sí gestionar las asignaciones universales, la obra social, beca escolar, lo que sea que permita mantener ese pibe con un nivel más o menos, que no tengas que ir al hospital a las 5 de la mañana. (Entrevista realizada a Juez de Familia, Bariloche, Provincia de Río Negro, diciembre de 2017).

Aun cuando no perciban un salario, las familias de acogimiento son acompañadas por una batería de recursos institucionales que, si bien modestos, permiten afrontar algunos gastos derivados del cuidado cotidiano de niños y niñas. La mayoría de las jurisdicciones otorga apoyo económico, ya sea para cubrir las necesidades del niño/a como para fines específicos (arreglo de vivienda, atención a la salud, útiles escolares, regalos de cumpleaños o navidad). A su vez, algunos programas ofrecen apoyo mediante módulos alimentarios, elementos de higiene para bebés, acompañantes, útiles escolares, asistencia psicológica o incorporación a prestaciones sanitarias. Complementariamente, en la mayor parte de las localidades, se implementan capacitaciones, encuentros de seguimiento y apoyo a través de grupos de whatsapp en los cuales están incluidas las y los agentes de los programas. Este último signo de cercanía, cuidado y acompañamiento es especialmente llamativo, en tanto ninguna instancia de esta naturaleza es creada para acompañar a las familias de origen y la flexibilidad e inmediatez de la comunicación telefónica suele ser reemplazada por la asistencia a las oficinas y las horas de espera. En contraste con ello, con las familias de acogimiento se organizan encuentros grupales, talleres, o bien como nos contaban en uno de los programas "grupos de autoayuda guiados por una psicóloga que les permita a las familias darse fuerzas entre ellas" (Entrevista a 
Coordinador de Programa estatal de Acogimiento Familiar, Provincia de Entre Ríos, noviembre de 2017). La idea rectora en la mayoría de los programas es la de "no largar solas" a las familias que realizan el acogimiento y ofrecerles apoyo constante.

Estos apoyos económicos se consideran sólo derivados de las necesidades de niños/as y adolescentes, de modo que los supuestos de los programas y las perspectivas de muchos de los funcionarios y trabajadores entrevistados dan por sentado que el trabajo de cuidado, realizado usualmente por las mujeres, debe derivar del altruismo con que se aborda la tarea, en una versión remozada de maternalismo conservador (Haney, 2002; Llobet; Villalta, 2018; Nari, 2004).

Los objetivos de algunos de los programas, sobre todo los más profesionalizados, que se identifican en los proyectos institucionales o resoluciones de creación que hemos relevado en nuestra investigación, así como el tipo de evaluación de los cuidados brindados por las familias de acogimiento que pudimos conocer a través de las entrevistas y charlas mantenidas con los profesionales que conforman los equipos técnicos de estos programas, muestran una marcada influencia de discursos psi, y también biologicistas y neurocientíficos sobre la infancia y la primera infancia en particular (Fonseca, 2011). De este modo, las "señales" e indicios para evaluar la calidad del cuidado brindado por las familias son ordenados en términos del adecuado crecimiento y desarrollo, y de criterios asociados al bienestar psi: el ceño que deja de estar fruncido, las manitos de los bebés que se abren, las sonrisas, la ganancia de peso resultan señales autoevidentes de lo positivo del cuidado singularizado. Asimismo, la posibilidad de cuidado es evaluada desde un punto de vista teleológico y normalizado, y los logros y beneficios del programa se visualizan en torno a mojones del desarrollo:

No se evalúa a las familias para ser padres, si no para situaciones que necesitan particular contención, por eso hay que evaluar la madurez emocional. (Entrevista realizada a Coordinador de Programa de Acogimiento Familia, Gral. Roca, Provincia de Río Negro, noviembre de 2017).

Es notable la diferencia, los chicos en acogimiento familiar hablan más temprano, son más expresivos. (Entrevista realizada a profesional del Programa estatal de Acogimiento Familiar, Ciudad Autónoma de Buenos Aires, diciembre de 2017). 
Las principales características que, en la dinámica concreta de su implementación, adquieren los programas, resultan en que el acogimiento familiar sea así un recurso para los organismos de protección (Llobet; Villalta, 2018; Villalta; Rojas; Gesteira, 2019) cuya meta es evitar tomar medidas en medios residenciales y propender al cuidado de tipo familiar, en lugar de un apoyo efectivo y transitorio para las familias de origen que no pueden sostener el cuidado de sus niños. También redundan en el hecho de que las familias de acogimiento cobran precedencia en la negociación de los criterios de inclusión de niños en los programas. Esto es, independientemente de los lineamientos formales del programa, para funcionarios y trabajadores es legítimo que las necesidades y preferencias de la familia acogedora sean una de las dimensiones mayormente tenida en cuenta por los programas para incorporar o no un niño/a o adolescente al acogimiento. Así la razón fundamental por la que principalmente los programas se destinan al cuidado de bebés o niños/as muy pequeños, es explicada en referencia a las preferencias y disponibilidad de las familias de acogimiento y al rechazo o resquemor de éstas de cuidar a adolescentes. En otras palabras, por más que en el diseño el programa sea formalmente para niños de 0 a 18 años, en la práctica solo se destina a niños de hasta 3 años, ya que las familias de acogimiento sólo aceptan ese rango etario y esto -como pudimos comprobar en las entrevistas realizadas- no suele ser objetado desde los programas. Finalmente, las interpretaciones de las necesidades infantiles y de las formas adecuadas y correctas de socialización infantil llevan a ponderar las necesidades de las familias de acogida como necesidades más válidas o legítimas que las de las familias de origen. En una interacción compleja entre cercanía social, cultural y moral entre agentes institucionales y familias de acogida, la eficacia de los estándares morales y materiales de la representación hegemónica sobre familia, los discursos psi sobre el cuidado y la niñez y los discursos sobre los derechos de los niños, se imbrican y resultan efectivos en la validación de necesidades y comportamientos considerados adecuados.

A partir de esta descripción, a continuación daremos cuenta de las formas de apoyo y arquitecturas de cuidado -esto es, las prestaciones, recursos y estrategias previstas institucionalmente- otorgadas a las familias de origen y las familias de acogimiento, y de las distintas valoraciones sobre los recursos, la moral y los afectos entre ambas, considerando las interpretaciones sobre el papel del dinero en el cuidado. 


\section{La reorganización y transformación del afecto familiar}

Las prácticas de cuidado, delegadas en la familia y el ámbito doméstico, son objeto de evaluación, control e intervención estatal. Estas actividades de cuidado conforman lo que Dolors Comas d'Argemir (2000) ha denominado economía del afecto, ${ }^{9}$ sosteniendo asimismo que el hecho de que el cuidado familiar se asocie a sentimientos y obligaciones morales ha contribuido a considerarlo como la solución óptima y más deseable frente a otras opciones (Comas d'Argemir, 2014). Al desplazarse al ámbito de las familias de acogida, el espacio se transforma y pasa a ser un espacio que, en tanto es capaz de imitar la intimidad familiar sin alterar las relaciones de parentesco, adquiere una confiabilidad mayor que la del cuidado brindado en la familia de origen. Esta confiabilidad, basada en las distinciones morales y de clase, hace a que la relación entre los programas estatales y las familias de acogida pueda limitarse al apoyo y a una suerte de control a distancia, que mantienen el ideal de privacidad y la "esfera privada" como ámbitos supuestamente por fuera del alcance del estado. Esto es, los apoyos brindados a las familias de acogida por los programas estatales pierden visibilidad ante la idea de privacidad familiar, y el propio trazado de tal frontera refuerza la noción de "mundos hostiles" (Zelizer, 1994) y contribuye también a validar la jerarquía moral sobre las familias de origen y a invisibilizar las materialidades de la reproducción social, que es interpretada solo en términos de disposición para el cuidado adecuado y amoroso.

Por su parte, las familias de acogimiento entrevistadas señalan que han lidiado con diferentes inconvenientes y situaciones emocionalmente complicadas cuando en contextos sociales e institucionales diversos (escuelas, plazas, clubes, etc,) queda en evidencia que los niños/as que cuidan no son sus hijos. Sorpresa por parte de quienes interactúan con ellos, preguntas por el dolor ante la pérdida que sobrevendrá cuando el niño/a sea retirado, o bien dudas sobre el altruismo o las intenciones que los han llevado a desempeñarse como familias de acogida son expresiones que reciben a menudo de sus propias

9 Con el término "economía del afecto", Comas d'Argemir (2000) señala el doble sentido de las actividades de cuidado tienen valor económico (lo que queda de manifiesto cuando las realiza el mercado o el estado) y también "economizan" gasto público. Queda de manifiesto además que el cuidado trasciende el ámbito familiar pues es un elemento imprescindible para la reproducción social (Carrasco; Borderías; Torns, 2011). 
redes vinculares o bien en la interacción con diferentes personas en salas de espera, plazas, o guarderías. Ello conduce a la elaboración de complejas operaciones subjetivas, morales, discursivas y emocionales por parte de las y los integrantes de estas familias de acogimiento, cuyo fin es restituir valor social a la forma de convivencia que emerge del acogimiento. La transitoriedad del vínculo de convivencia, la mutación del amor paterno-filial en otro tipo de amor, y la deseabilidad de la perdurabilidad más allá de la convivencia del vínculo social creado entre acogientes y acogidos, representan desafíos a los que las familias de acogimiento se enfrentan cotidianamente.

Las tensiones entre afecto y cuidado son así interpretadas y reorganizadas a partir de la matriz simbólica provista por la naturaleza transitoria y no filiatoria del vínculo. La moralización del cuidado tiende a negar u ocultar las tensiones y los conflictos (Comas d'Argemir; Marre; San Román, 2016), pero en el caso del acogimiento familiar esas tensiones son eficientemente externalizadas a la naturaleza del propio dispositivo y, en no pocos casos, atribuidas a la familia de origen del niño en acogimiento. Así, los sentimientos negativos, las angustias y las tensiones propias del cuidado y de la naturaleza temporal del mismo son organizadas a través de las narrativas del abandono y de la ineficiencia de la burocracia.

Les pedí que cuando la restitución fuera una realidad, me dieran tiempo para procesarlo y despedirme: me sentiría re utilizada, porque no estás cuidando un kilo de hierba... más allá de que yo sepa que es transitorio y que la bebé no es mi hija, lo cierto es que se ponen en juego un montón de cosas, y no es que me tengan que cuidar a mí, pero un gesto mínimo espero. (Entrevista realizada a integrante de una familia de acogimiento familiar, Programa estatal de Acogimiento Familiar, Provincia de Neuquén, diciembre de 2017).

El trazado de fronteras entre rédito económico, cuidado y amor contribuye a edificar el valor social de la infancia (Zelizer, 1994), y resulta también una dimensión importante en el establecimiento de jerarquías sociales de género adjudicando a la figura de la madre un rol central en la organización de las protecciones sociales (Fraser; Gordon, 1994; Gordon, 1994). En efecto, la distinción entre el carácter moralmente justificado de ciertas necesidades y no de otras, el valor moral de algunos sujetos de protección y no de otros, también 
se construye a partir del trazado de tal frontera. A la vez, muchos agentes disputan estos sentidos, y señalan que el carácter oneroso del cuidado puede ser motivo de la limitada convocatoria de familias de acogimiento. Complementariamente, el énfasis en el carácter altruista o solidario del acogimiento sesga la balanza prioritariamente hacia familias de extracción religiosa o vinculadas con tareas de docencia y cuidados, y también hacia las familias que posean un buen pasar en términos económicos. Estas distancias de clase no resultan invisibles ni dejan de ser problematizadas por algunos trabajadores, quienes plantean dudas sobre tal distancia social entre las familias de sectores acomodados y los niños y niñas en acogimiento.

Las expectativas respecto de los espacios en que tienen que vivir los niños se vinculan con las transformaciones del estatus moral y el valor social de los niños. En la medida en que niños y niñas fueron "sacralizados", comenzaron a ser valorados, ya no por su contribución a la reproducción social y al ingreso familiar, sino por su invaluable aporte emocional. De tal manera, los intercambios económicos vinculados con los niños, y su valoración utilitaria fueron moral y normativamente condenados (Zelizer, 1994) y debió así garantizarse que la frontera entre los mundos hostiles del amor y el dinero no se rompa.

El "afán de lucro" de cuidadoras, madres y adultos en general, configura uno de los límites e intolerables capaz de reorganizar las relaciones familiares y el emplazamiento simbólico y físico de los niños en el marco de la intervención de protección de derechos. Complementariamente, el "altruismo" y la construcción del ámbito privado como un espacio exento de cálculos económicos contribuyen a la reproducción del cuidado como una tarea sólo derivada del amor y cuyas expresiones materiales deben ser subsidiarias, so pena de corromper y cubrir con una sombra de duda la calidad de tal cuidado, en lo que Zelizer ha señalado como los mundos hostiles del amor y el dinero. Esta oposición entre altruismo e interés configura un par de términos clave, en el sentido de Fraser y Gordon (1994), esto es, capaces de articular sentidos socialmente disponibles en torno a jerarquías de género y de clase.

$\mathrm{Si}$ la frontera entre amor y lucro es considerada suficiente para las familias de acogida al no mediar pago en dinero, el esfuerzo exigido a las familias de origen para demostrar que no "usufructúan o explotan" a sus hijos e hijas es mayor y constante. Asimismo, si las llamadas pidiendo apoyo o recursos por parte de las familias de acogimiento son atendidas como legítimas y adecuadas, y son 
interpretadas como un signo de su abocamiento a proveer cuidados apropiados al niño o niña bajo su protección, cuando comportamientos similares son desarrollados por parte de las familias biológicas tienden a ser considerados como signos de una demanda excesiva o propios de familias "muy querellantes", y una muestra más de lo inadecuado del vínculo. En otras palabras, la disponibilidad para acompañar a las familias de acogimiento encuentra, en espejo, una sospecha permanente hacia las familias de origen. Esta sospecha que pesa sobre estas familias, una vez tomada la medida excepcional, raramente es revertida e invade la percepción de cada detalle. Las dos referentes de un novísimo programa de acogimiento de un municipio del área metropolitana de Buenos Aires expresaron: "Las familias de origen no vienen a las vinculaciones, no muestran ningún esfuerzo. Les mostramos una foto del niño en la entrevista y fue como si nada, ninguna emoción". Por un lado, la complejidad de los traslados -desde la mala distribución del transporte público hasta el peso de su costo en el marco de los magros ingresos de las familias de sectores populares-y la complejidad emocional de la situación -asistir a una oficina que se halla en el mismo edificio en el que se tomó la medida excepcional y la inseguridad y temor que pueden sentir frente a la "otra" familia- no son problematizadas por estas agentes quienes además realizan esta operación de totalización -que crea una serie y generaliza la actitud de "las familias de origen"-a partir de uno de cinco de los casos que en total tuvieron en el programa de acogimiento recientemente inaugurado.

Por su parte, las familias de origen disponen solo de un lapso de seis meses para modificar la situación que condujo a la separación del niño o niña y a su inclusión en un programa de acogimiento familiar. Los agentes de estos programas desconocen qué tipo de acompañamiento se realiza en ese ínterin, aunque en la mayoría de los casos el mismo se traduce en alguna forma de "puerta de entrada" al estado: gestión de los inaccesibles turnos para asistencia en salud mental, algún que otro apoyo puntual y, fundamentalmente, la supervisión y el seguimiento para evaluar si modificaron sus hábitos. En los casos en que los agentes que toman la medida excepcional lo consideren, se desarrollan estrategias de revinculación, en las sedes de los programas de acogimiento o en espacios públicos, siempre en presencia del personal del programa y nunca de la familia de acogimiento. La revinculación es también una escena de supervisión, lejana a la posibilidad de apoyar a las madres a producir novedosas modalidades de vínculo: 
La sala donde se realizan las revinculaciones es una oficina blanca con una puerta y una ventana. Tiene un escritorio con una silla para la profesional y dos para las familias. Ocupa la mitad de la sala una mesita infantil, hay juguetes y una alfombra - rompecabezas de goma eva. Cuando llegué estaban esperando a un niño para una revinculación. La mamá estaba siendo entrevistada por la trabajadora social en la sala, y cuando el niño llegó, lo llevaron a la sala y la trabajadora social salió, dejaron la puerta abierta y ella permaneció parada en el pasillo, mirando desde afuera. Una vez que vio que "todo estaba bien", la trabajadora social se desplazaba por el pasillo conversando con otros colegas respecto a distintas cuestiones laborales, y cada tanto volvía a la puerta. (Observación de campo, Rio Grande, Provincia de Tierra del Fuego, febrero de 2018).

En otras palabras, el argumento que tratamos de colocar es que son notables las dificultades de los programas para reconocer la profunda y compleja imbricación entre las materialidades, afectividades y moralidades del cuidado de los niños. Las narrativas institucionales disponibles para las familias de origen son así limitadas y restrictivas. Las dificultades materiales cobran dimensiones morales. Si las madres no logran construirse como "confiables" (Santillán, 2009), deberán transitar un empinado camino para lograr revertir las decisiones institucionales. Reversión que es más vale inusual. Como planteaba una mujer cuya hija había sido ubicada en una familia de acogimiento luego de que se adoptara una medida excepcional:

Yo no quería que ella se fuera con otra familia, pero me tengo que dejar llevar. No tengo plata ni abogados para sacarla de ahí. (Madre de origen de una niña ubicada en una familia de acogimiento familiar, Provincia de Neuquén, diciembre de 2017).

\section{Consideraciones finales}

La centralidad de la tensión entre amor y dinero, y cuidado y trabajo, así como su capacidad para organizar las prácticas de distribución de responsabilidad sobre los niños, hace a las políticas de acogimiento un analizador relevante de transformaciones en curso, debido a que muestran procesos de redefinición de las relaciones entre estado, mercado y familias, y la producción de exclusiones 
y jerarquías sociales y de género. En efecto, al priorizar un amor desinteresado y un cuidado "de calidad" moralizado, los requisitos materiales y organizacionales del cuidado son invisibilizados, permitiendo que los bienes y los niños y niñas circulen en dirección a las familias más aventajadas que no solo cuentan con más recursos, sino también son beneficiarias de una política más comprensiva que aquella que se dirige a las familias más frágiles.

La no conciliación de ambas esferas, vistas además como antagónicas (amor y dinero, cuidado y salario, relaciones afectivas y relaciones contractuales), opera como un límite conceptual al momento de pensar estrategias y alternativas. A la vez, naturaliza representaciones y prácticas cuyos efectos en las desigualdades de género y clase han sido profusamente estudiadas. Además de ello, pareciera que las familias de origen sólo pueden realizar demandas y/o peticiones que se ajusten a las "necesidades" de los niños legitimadas como tales, ya que solo así tales demandas resultan audibles. La complejidad de la trama de necesidades, que incluye tanto la posibilidad de reaprender las prácticas de crianza como los apoyos materiales, emocionales y sociales para el cuidado no aparecen en el espectro de posibilidades de intervención movilizadas por los programas. Ello restringe el espacio de maniobra que tienen, en tanto no les permite conectar las necesidades complejas de los distintos miembros del grupo, dado que desde los agentes institucionales, muchas veces se opone el interés del niño al de su familia de origen.

En este sentido, tal como postula Claudia Fonseca, las microprácticas institucionales tanto como las arquitecturas del cuidado para producir la transferencia de niños inciden y reactualizan procesos de discriminación simbólica contra los pobres en el marco de las políticas de protección de derechos. De este modo, en la interrelación entre prácticas locales, legislación nacional, y política global van configurándose como legítimos determinados valores familiares. Esta perspectiva, inspirada en la noción de "reproducción estratificada" (Colen, 1995 apud Fonseca, 2002), se concentra en el modo en que diferentes repertorios culturales se entretejen, chocan o se complementan entre sí, de acuerdo con circunstancias históricas particulares. El escrutinio de la diferencia cultural es, sobre todo, inseparable de las consideraciones de desigualdad política y social que provoca que ciertos valores sean vistos como superiores a otros. 
Por otro lado, también es posible señalar que antes que constituirse en autoridades complementarias, como señalara Vianna respecto del poder tutelar, los organismos y agentes estatales despliegan prácticas de acompañamiento y soporte a unas familias, toleran algunas prácticas familiares, mientras que despliegan acciones punitivas y persecutorias respecto de otras (Villalta, 2013), a partir de la negociación de sentidos variables y estratégicos en torno al cuidado, el dinero y las emociones.

Finalmente, nos interesa dejar planteado que las interpretaciones de necesidades -emocionales, subjetivas y económicas- que articulan las políticas de acogimiento constituyen la trama de negociaciones en las que los sentidos y alcances de los derechos son redefinidos, limitando la capacidad de los sectores populares de usarlos en su favor. De este modo, como hemos visto, a partir de los valores que se movilizan en la práctica concreta del acogimiento familiar se tienden a privilegiar respuestas individualizadas y responsabilizantes. La muy diversa alocación de recursos y apoyos para las distintas familias involucradas, así como la diversa significación que adquieren los mismos en relación a los cuidados de niños y niñas, se vincula con el trabajo institucional de redefinición de cuidados, vínculos y responsabilidades familiares. Las cuidadoras de los sectores de origen de los niños no sólo tienen un acceso limitado a los recursos para desplegar el cuidado, sino que además el sistema de fronteras, intolerables y jerarquías morales desestima la posibilidad de conectar demandas (Haney, 2002) y de lograr así construir mayores posibilidades de cuidar. No obstante, en las experiencias concretas del acogimiento familiar también es posible vislumbrar la existencia de otros sentidos que, en ocasiones, pugnan por prevalecer. Valores asociados al pluriparentesco y a la complementariedad en el cuidado, y que se oponen a la sustitución, reemplazo y corte abrupto de lazos, son también accionados en algunas experiencias en las que las demandas, necesidades y capacidades de las familias de origen no son por completo desestimadas sino que resultan integradas a la dinámica del cuidado. En suma, si al nivel del diseño, de la alocación de recursos y de la implementación de imperativos normativos estos programas se ajustan a lógicas individualizantes, en el nivel de la dinámica cotidiana y de la construcción de lazos hay siempre espacios para el despliegue de tácticas que disputen la hegemonía. 


\section{Referencias}

BALAGOPALAN, S. Why historicize rights-subjectivities? Children's rights, compulsory schooling, and the deregulation of child labor in India. Childhood, [s. l.], v. 26, n. 3, p. 304-320, 2019. DOI: https://doi.org/10.1177/0907568219856077.

BARNA, A. La gestión de la infancia entre lo local y lo global: una etnografía sobre intervenciones destinadas a "restituir derechos de niños" en dispositivos estatales en el marco de las Leyes de Protección Integral. 2015. Tesis (Doctorado en Antropología Social) - Facultad de Filosofía y Letras, Universidad de Buenos Aires, Buenos Aires, 2015.

BARRANCOS, D. Anarquismo, educación y costumbres... en la Argentina de principios de siglo. Buenos Aires: Contrapunto, 1999.

BITTENCOURT RIBEIRO, F. Acolhimento de famílias e modos de apoio à (pluri) parentalidade. Scripta Nova, Barcelona, v. 16, n. 395, 2012. Disponible en: http://www. ub.edu/geocrit/sn/sn-395/sn-395-4.htm. Acceso: 5 oct. 2020.

CADORET, A. Parenté plurielle: anthropologie du placement familial. Paris: L'Harmattan, 1995.

CADORET, A. Rubrique - Enfants placés, parents suppléants: filiation et affiliation. Informations sociales, [s. l.], v. 131, n. 3, p. 120-121, 2006.

CARRASCO, C.; BORDERÍAS, C.; TORNS, T. (ed.). El trabajo de cuidados: historia, teoría y políticas. Madrid: Libros de la Catarata, 2011.

CHENEY, K. Killing them softly? Using children's rights to empower Africa's orphans and vulnerable children. International Social Work, [s. l.], v. 56, n. 1, p. 92-102, 2013.

CIORDIA, C.; VILLALTA, C. Procedimientos judiciales y administrativos de adopción de niños. Confrontación de sentidos en la configuración de un "medio familiar adecuado". Etnográfica, Lisboa, v. 16, n. 3, p. 435-460, 2012.

COMMAILlE, J.; MARTIN, C. Les enjeux politiques de la famille. Paris: Bayard, 1998.

COMAS D'ARGEMIR, D. Mujeres, familia y estado del bienestar. In: VALLE, T. del (ed.). Perspectivas feministas desde la antropología. Barcelona: Ariel, 2000. p. 187-204.

COMAS D’ARGEMIR, D. Los cuidados y sus máscaras. Retos para la antropología feminista. Mora, Buenos Aires, n. 20, p. 167-182, 2014.

COMAS D'ARGEMIR, D.; MARRE, D.; SAN ROMÁN, B. La regulación política de la familia. Ideología, desigualdad y género en el Plan Integral de Apoyo a la Familia. Política y Sociedad, [s. l.], v. 53, n. 3, p. 853-877, 2016. 
COSSE, I. Argentine mothers and fathers and the new psychological paradigm of child-rearing (1958-1973). Journal of Family History, [s. l.], v. 35, n. 2, p. 180-202, 2010.

DARRÉ, S. Maternidad y tecnologías de género. Buenos Aires: Katz, 2013.

DONZELOT, J. La policía de las familias. Valencia: Pretextos, 1990.

FASSIN, D. Moral economies revisited. Annales. Histoire, Sciences Sociales, Paris, v. 64, n. 6, p. 1237-1266, 2009.

FEDERICI, S. El patriarcado del salario: críticas feministas al marxismo. Buenos Aires: Tinta Limón, 2018.

FONSECA, C. Caminos de la adopción. Buenos Aires: Eudeba, 1998.

FONSECA, C. Inequality near and far: adoption as seen from the Brazilian favelas. Law \& Society Review, [s. l.], v. 36, n. 2, p. 101-134, 2002.

FONSECA, C. Aventuras familiares: do acolhimento à adoção. In: FONSECA, C.; SCHUCH, P. (org.). Políticas de proteção à infância: um olhar antropológico. Porto Alegre: UFRGS Editora, 2009. p. 273-294.

FONSECA, C. Tecnologías globales de la moralidad materna: políticas de educación para la primera infancia en Brasil contemporáneo. In: COSSE, I. et al. (comp.). Infancias: políticas y saberes en Argentina y América Latina (siglos XIX-XX). Buenos Aires: Editorial Teseo, 2011. p. 175-204.

FRASER, N.; GORDON, L. "Dependency" demystified: inscriptions of power in a keyword of the welfare state. Social Politics, [s. l.], v. 1, n. 1, p. 4-31, Spring 1994.

GORDON, L. Pitied but not entitled: single mothers and the history of welfare 18901935. New York: The Free Press, 1994.

HANEY, L. Inventing the needy: gender and the politics of welfare in Hungary. Berkeley: University of California Press, 2002.

HANEY, L.; POLLARD, L. (ed.). Families of a new world: gender, politics and state development in a global context. London: Routledge, 2003.

JELIN, E. Pan y afectos: la transformación de las familias. Buenos Aires: Fondo de Cultura Económica, 1998.

LEINAWEAVER, J. Los niños ayacuchanos: una antropología de la adopción y la construcción familiar en Perú. Lima: Institutos de Estudios Peruanos, 2009.

LEINAWEAVER, J. El desplazamiento infantil: las implicaciones sociales de la circulación infantil en los Andes. Scripta Nova, Barcelona, v. 16, n. 395, 2012. Disponible en: http://www.ub.edu/geocrit/sn/sn-395/sn-395-13.htm. Acceso: 5 oct. 2020. 
LLOBET, V. Fábricas de niños: las instituciones en la era de los derechos. Buenos Aires: Novedades Educativas, 2009.

LLOBET, V. La producción de necesidades infantiles en las políticas de protección de derechos. Psicologia em Estudo, Maringá, v. 19, n. 3, p. 369-380, jul./set. 2014.

LLOBET, V. El trabajo emocional como trabajo político: niños/as y jóvenes en los márgenes del Estado. [S. l: s. n.], 2019. Ponencia presentada en LASA Boston.

LLOBET, V.; MILANICH, N. La maternidad y las mujeres de sectores populares en las Transferencias Condicionadas de Ingresos. Un aporte al debate sobre el cuidado y las relaciones de género. Zona Franca: revista del Centro de Estudios Interdisciplinarios sobre Mujeres, [s. l.], año 22, n. 23, p. 58-69, 2014.

LLOBET, V.; VILLALTA, C. Relevamiento y sistematización de Programas de Cuidado Alternativo en ámbito familiar. Buenos Aires: Unicef Argentina, 2018.

LLOBET, V.; VILLALTA, C. (coord.). De la desjudicialización a la refundación de los derechos: transformaciones en las disputas por los derechos de los niños y las niñas (2005-2015). Buenos Aires: Teseo Press, 2019.

LUGONES, M. G. Obrando en autos, obrando en vidas: formas y fórmulas de Protección Judicial en los tribunales Prevencionales de Menores de Córdoba, Argentina, a comienzos del siglo XXI. Rio de Janeiro: Laced/UFRJ, 2012.

MAGISTRIS, G. El gobiero de la infancia en la era de los derechos. 2016. Tesis (Doctorado en Ciencias Sociales) - Facultad de Ciencias Sociales, Universidad de Buenos Aires, Buenos Aires, 2016.

MARRE, D. Los silencios de la adopción en España. Revista de Antropología Social, [s. l.], v. 18, p. 97-126, 2009.

MILANICH, N. Illegitimacy and illegitimates. In: HECHT, T. (ed.). Minor omissions: children in Latin American history and society. Madison: University of Wisconsin Press, 2002. p. 72-101.

NARI, M. Las políticas de la maternidad y maternalismo político, Buenos Aires, 1890-1940. Buenos Aires: Biblos, 2004.

ROSEN, R. Poverty and family troubles: mothers, children, and neoliberal "antipoverty” initiatives. Journal of Family Issues, [s. l.], v. 40, n. 16, p. 2330-2353, 2019.

SANTILLÁN, L. Antropología de la crianza: la producción social de 'un padre responsable' en barrios populares del Gran Buenos Aires. Etnográfica, Lisboa, v. 13, n. 2, p. 265-289, 2009. 
SOUZA LIMA, A.; MACEDO E CASTRO, J. Notas para uma abordagem antropológica da(s) política(s) pública(s). Anthropologicas, Recife, v. 26, n. 2, p. 17-54, 2015.

STOLER, A. Carnal knowledge and imperial power: race and the intimate in colonial rule. Oakland: University of California Press, 2007.

THOMPSON, E. P. Los orígenes de la ley negra: un episodio de la historia criminal inglesa. Buenos Aires: Siglo XXI, 2010.

TORRADO, S. Historia de la familia en la Argentina moderna (1870-2000). Buenos Aires: Ediciones de la Flor, 2003.

UNICEF; SECRETARÍA NACIONAL DE NIÑEZ, ADOLESCENCIA Y FAMILIA. Situación de niñas, niños y adolescentes sin cuidados parentales en la República Argentina: relevamiento nacional: actualización 2017. Buenos Aires: Unicef: Senaf, 2018. Disponible en: https://www.argentina.gob.ar/sites/default/files/cuidados.parentales.2017_ fnl26-11_2.pdf. Acceso: 5 oct. 2020.

URIARTE BÁLSAMO, P.; FONSECA, C. Paradoxos inerentes nos programas de atendimento para crianzas em risco: o caso do Programa de Lares Substitutos - RS. In: FONSECA, C.; SCHUCH, P. (org.). Políticas de proteção à infância: um olhar antropológico. Porto Alegre: UFRGS Editora, 2009. p. 145-169.

VIANNA, A. Quem deve guardar as crianças? Dimensões tutelares da gestão contemporânea da infância. In: SOUZA LIMA, A. C. (org.). Gestar e gerir: estudos para uma antropologia da administração pública no Brasil. Rio de Janeiro: Relume Dumará, 2002. p. 271-312.

VILLALTA, C. La conformación de una matriz interpretativa: la definición jurídica del abandono y la pérdida de la patria potestad. In: LIONETTI, L.; MíGUEZ, D. (comp.). Las infancias en la historia argentina: intersecciones entre prácticas, discursos e instituciones (1880-1960). Rosario: Prohistoria, 2010. p. 71-93.

VILLALTA, C. Entregas, adopciones y dilemas en el campo de organismos destinados a la infancia. Revista Estudos Feministas, Florianópolis, v. 19, n. 1, p. 103-123, 2011.

VILLALTA, C. Entregas y secuestros: el rol del Estado en la apropiación de niños. Buenos Aires: Editorial Del Puerto, 2012.

VILLALTA, C. Un campo de investigación: las técnicas de gestión y los dispositivos jurídico-burocráticos destinados a la infancia pobre en la Argentina. Civitas, Porto Alegre, v. 13, n. 2, p. 245-268, 2013.

VILLALTA, C. Ampliando derechos para los niños y las niñas, agilizando procedimientos para los “menores”. Mora, Buenos Aires, v. 21, n. 1, p. 187-193, 2015. 
VILLALTA, C.; ROJAS, S.; GESTEIRA, S. ¿Quién y cómo cuida los niños y las niñas? Disputas sobre la instalación del acogimiento familiar como política pública en la Argentina. In: LLOBET, V.; VILLALTA, C. (coord.). De la desjudicialización a la refundación de los derechos: transformaciones en las disputas por los derechos de los niños y las niñas (2005-2015). Buenos Aires: Teseo Press, 2019. p. 483-519.

ZELIZER, V. Pricing the priceless child: the changing social value of children. Princeton: Princeton University Press, 1994.

Recebido: 08/10/2020 Aceito:6/7/2021 | Received:10/8/2020 Accepted: 7/6/2021 